\title{
Breast Cancer in Nepal: Current status and future directions (Review)
}

\author{
MOHAN GIRI $^{1 *}$, MAMATA GIRI $^{1 *}$, RABIN JUNG THAPA $^{2}$, BIBHUTI UPRETI $^{1}$ and BIJAY PARIYAR ${ }^{3}$ \\ Departments of ${ }^{1}$ Respiratory Medicine, ${ }^{2}$ Dermatology and Venerology and ${ }^{3}$ Orthopedics, \\ The First Affiliated Hospital of Chongqing Medical University, Chongqing 400016, P.R. China
}

Received January 19, 2018; Accepted February 1, 2018

DOI: $10.3892 /$ br.2018.1057

\begin{abstract}
Breast cancer is the second most common malignancy among Nepalese women. Breast cancer places a substantial burden on the Nepalese healthcare system, but information regarding the number of women living with breast cancer is not well recorded. In countries with lower levels of resources such as Nepal, breast cancers are commonly diagnosed at late stages and women may receive inadequate treatment, pain relief or palliative care. Socioeconomic disparities and insufficient financial resources hinder prevention of breast cancer in Nepal. The current review provides an overview of the burden of breast cancer, of risk factors associated with breast cancer, and of screening and treatment modalities for breast cancer in Nepal. Additionally, this review highlights the current awareness of breast cancer among Nepalese women and prevention strategies for breast cancer.
\end{abstract}

\section{Contents}

1. Introduction

2. Burden of breast cancer in Nepal

3. Risk factors

4. Current screening of breast cancer in Nepal

5. Treatment modalities of breast cancer in Nepal

6. Breast cancer awareness and future directions

7. Conclusion

Correspondence to: Dr Mohan Giri, Department of Respiratory Medicine, The First Affiliated Hospital of Chongqing Medical University, 1 Yi Xue Yuan Lu, Yuzhong, Chongqing 400016, P.R. China

E-mail: mohangiri175@yahoo.com

*Contributed equally

Key words: breast cancer, Nepal, prevention, risk factors, treatment

\section{Introduction}

Breast cancer is a major public health problem in developing countries. The American Cancer Society has estimated that approximately 1,735,350 new cases of cancer and 609,640 cancer-related fatalities will occur in the United States (US) in 2018, including 266,120 new cases of invasive breast cancer (1). Furthermore, in the US the healthcare costs of cancer in 2010 surpassed $\$ 124.5$ billion (USD), with this figure estimated to reach $\$ 157.8$ billion by 2020 , of which the highest costs will be for breast cancer (2). A study by Youlden et al (3) in 2014 revealed that breast cancer was the most prevalent cancer and fourth leading cause of cancer-related mortality among women in Asia. In Nepal, according to the data from seven major cancer service hospitals in 2012, breast cancer was the second most common cancer among women, after cancer of the cervix $(4,5)$. The risk of breast cancer increases exponentially up to the age of menopause, and increases at a slower rate thereafter (6). Early detection of breast cancer markedly increases the probability of successful treatment. Nevertheless, there is a need for culturally appropriate, targeted intervention to promote preventive strategies and enhance knowledge and awareness regarding breast cancer. A similar previous review has been conducted in India by Agarwal and Ramakant (7), which concluded that breast cancer was the most prevalent cancer in Indian women living in urban regions, while awareness of the symptoms, early diagnosis and screening tests for breast cancer was notably poor. Previous studies have demonstrated that there are sub-optimal levels of breast cancer awareness, knowledge of risk factors and screening among Nepalese women $(8,9)$.

Breast cancer is a substantial social and economic burden in Nepal. Prevention has been demonstrated to be among the most effective long-term strategies to lessen the increasing chronic disease burden (7). However, due to socioeconomic disparities and insufficient financial resources, to date, the prevention of breast cancer has not been well conducted in Nepal (4). As a developing nation, Nepal is faced with several challenges with regards to the care of patients with breast cancer with inadequate funding; the uneven distribution of resources and services; inadequate numbers, training and distribution of health-care personnel and equipment; and a lack of adequate care for many populations based on 
socioeconomic and geographic factors (4). In the present review, the epidemiological characteristics, risk factors and current breast cancer awareness and screening efforts in Nepal are summarized. Additionally, alternative ways to improve the prevention of breast cancer in Nepal are discussed.

\section{Burden of breast cancer in Nepal}

According to GLOBOCAN 2012 (10), an estimated 1,700 new breast cancer cases were diagnosed in Nepal in 2012, with an age standardized rate (ASR) of 13.7 new cases per 100,000 omen, while 870 fatalities in women occurred, with an ASR of 7.2 fatalities per 100,000 women (Fig. 1). Figure 1 compares these data with those of other countries (10) including India (ASR incidence: 25.8, ASR mortality: 12.7), Pakistan (ASR incidence: 50.3, ASR mortality: 25.2), Bangladesh (ASR incidence: 21.7, ASR mortality: 11.1), the US (ASR incidence: 92.9, ASR mortality: 14.9) and the United Kingdom (ASR incidence: 95 and ASR mortality: 17.1). While still noteworthy, the ASRs were markedly lower in Asian than in Western countries, which may be due to rapid economic growth and urbanization, and rises in the population's socioeconomic status in developed countries (10). Population-based cancer registries are considered the best option for measuring indicators of cancer (11). Although a population-based national cancer registry does not exist in Nepal, there is a multi-institution hospital-based registry. The most common age of breast cancer patients has been reported to be 40-50 years in various population-based studies performed in different parts of Nepal $(12,13)$, among which a marked proportion of Nepalese breast cancer patients were younger than 50 years of age.

\section{Risk factors}

The risk of breast cancer increases with age. Risk factors associated with breast cancer (Table I), including early menarche (before age 12), late menopause (after 55 years of age), nulliparity or first child birth after the age of 30 years, are similar in Asian and western populations, except for hormone replacement therapy (HRT), which is an important risk factor in Caucasians but not in Asian women (14-17). The adoption of the westernized lifestyle of delayed childbearing, reduced breastfeeding and other westernized dietary and lifestyle patterns has a significant impact on breast cancer risk and prognosis among Asian women (18). Studies have revealed that breast cancer in premenopausal Nepalese patients was associated with late menarche ( $>14$ years), early first full-term pregnancy (before 40 weeks of pregnancy) and a longer duration of breastfeeding, when compared with Japanese subjects $(19,20)$. Family history is also an important risk factor for breast cancer, but there is no association between family history of breast cancer and breast cancer severity or associated mortality (21). A family history of female breast cancer in first degree relatives is reportedly rare in a Nepalese context (19). Meanwhile, studies have identified that high dietary fat, excessive alcohol intake, hormone replacement therapy, smoking and exposure to radiation may be associated with an increased risk of breast cancer in Nepalese women among other nationalities $(22,23)$.

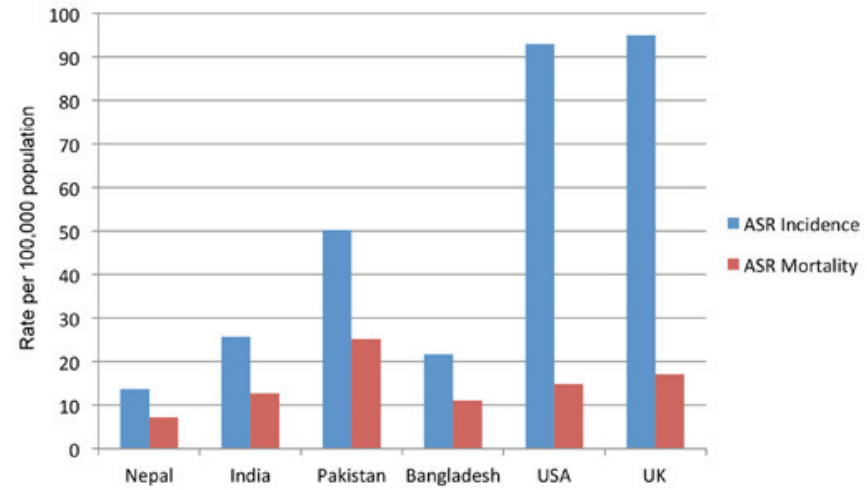

Figure 1. Comparison of ASRs of incidence and mortality related to breast cancer in women in Nepal and other countries. ASR is averaged per 100,000 women. ASR, age standardized rate.

\section{Current screening of breast cancer in Nepal}

Similar to India (7), no national or regional breast cancer screening program exists in Nepal. Early detection of breast cancer serves a leading role in the reduction of breast cancer mortality $(7,21)$. Among different methods of screening recommended for breast cancer (Table II), breast self-examination (BSE) and clinical breast examination are the most effective steps for detecting breast cancer at an early stage (24). Furthermore, studies have demonstrated that mammography screening may reduce breast cancer mortality risk by $20 \%$, and thus screening may be regarded as the optimal method to prevent breast cancer $(24,25)$. At present, parallel to India, mammography is not available in a large number of public and private hospitals in towns as a diagnostic service for women $(7,26)$. A study in Nepal in 2008 demonstrated that BSE could be regarded as tool to aid primary prevention strategies for breast cancer (23). High-resolution ultrasound may afford improved breast cancer detection and differentiation; however, ultrasound is generally only used if a suspicious lesion is detected by mammography (27). Kelly et al (28) revealed that automated whole breast ultrasound resulted in significantly improved cancer detection compared with mammography alone. For BRCA mutation carriers and their first-degree relatives, the American Cancer Society (ACS) recommended breast magnetic resonance imaging (MRI) screening as an adjunct to mammography (29). As a country with limited resources, in Nepal the number of MR imaging units is markedly low in proportion to the number of hospitals and its population. Thus, for early detection of breast cancer in Nepal, awareness and breast self-examination should be promoted through newspapers, electronic media, as well as through health personnel in various settings (7).

\section{Treatment modalities of breast cancer in Nepal}

National breast cancer management protocols in Nepal are at an early stage. To date, the treatment of breast cancers in Nepal has been decided by factors including financial situation, accessibility to the appropriate institution and social condition of the patient (4). There are number of clinical courses for treating breast cancer (Table III), which depend on its type and stage. Modified radical mastectomy, simple 
Table I. Risk factors of breast cancer in Nepal.

\begin{tabular}{lc}
\hline Risk factor & Refs. \\
\hline $\begin{array}{l}\text { Late menarche }(>14 \text { years of age) } \\
\text { Nulliparity or late age at first birth }\end{array}$ & $(19,20)$ \\
$(>35$ years of age) & $(19,20)$ \\
Longer duration of breastfeeding & $(19,20)$ \\
Family history & $(19,21)$ \\
Smoking & $(22,23)$ \\
Excessive alcohol consumption & $(22,23)$ \\
(>8 units per week) & \\
Consumption of fat $(\mathrm{BMI} \geq 30 \mathrm{~kg} / \mathrm{m} 2)$ & $(22,23)$ \\
Exposure to radiation & $(14,23)$ \\
Hormone replacement therapy & $(15,22)$ \\
\hline
\end{tabular}

BMI, body mass index.

Table II. Screening methods for breast cancer in Nepal.

\begin{tabular}{lc}
\hline Method & Refs. \\
\hline Breast self-assessment & $(23,24)$ \\
Mammography & $(23-25)$ \\
Ultrasound & $(23-28)$ \\
Magnetic resonance imaging & $(23,29)$ \\
\hline
\end{tabular}

Table III. Treatment options for breast cancer in Nepal.

\begin{tabular}{l}
\hline Modality \\
\hline Surgery \\
Total radical mastectomy \\
Simple mastectomy/toilet mastectomy \\
BCS \\
Radiotherapy \\
To the breast following BCS \\
To the chest wall following radical mastectomy \\
Systemic therapy \\
Tamoxifen \\
Anthracycline combination \\
Palliative treatment: \\
Opioid analgesics \\
Non Steroidal Anti Inflammatory Agents \\
Glucocorticoids \\
Systemic administration of radioisotopes
\end{tabular}

BCS, breast conservative surgery.

mastectomy or toilet mastectomy are frequently practiced surgical techniques for the management of breast cancer in Nepal (30). Of preference may be breast conserving surgery
(BCS) followed by moderate-dose radiation therapy to eradicate any microscopic residual disease, which provides the survival equivalent of mastectomy, reduced cosmetic impact, less anxiety and depression and improved body image (31). Studies have demonstrated that prior to 2009, trends of BCS in Nepal ranged from 6 to $15 \%$, while the most common operation performed was modified radical mastectomy $(4,19)$. Alike Indian populations, few Nepalese patients are offered post-mastectomy reconstruction, and only a small proportion of those offered accept to have it performed, probably due to the reconstructive procedure being seen as an unnecessary financial burden (7,30). The presence of axillary lymph node involvement is among the most important prognostic factors in breast cancer patients (32). Surgical staging of breast cancer by axillary lymph node dissection remains to be frequently practiced by many hospitals in Nepal (19); however, axillary lymph node dissection is increasingly being replaced by the sentinel node procedure, and sentinel node biopsy is now regarded as the standard procedure for axillary staging of breast cancer (33).

Radiotherapy reduces local recurrence rates and increases breast cancer-specific survival in patients with early-stage breast cancer. Previous data indicates that the survival of breast cancer patients is improved by post-mastectomy radiotherapy (34). Radiotherapy is typically administered to the breast following conservation surgery and may be administered to the chest wall following mastectomy to complete local treatment (35). A study by Sapkota et al (36) in 2016 identified that radiotherapy was among the most commonly used modalities for the treatment of breast cancer in Nepal

Systemic treatment of breast cancer includes the use of cytotoxic, hormonal and immunotherapeutic agents (37). Although metastatic breast cancer is unlikely to be cured, with the introduction of newer systemic therapies, notable improvements in survival have been observed in patients with breast cancer (37). The main aim of systemic therapies is prolongation of survival, alleviation of symptoms and improvement of quality of life. Tamoxifen has been used for more than 30 years to treat hormone receptor-positive breast cancer. A previous meta-analysis revealed that adjuvant therapy with tamoxifen resulted in a significant decrease in breast cancer mortality rate, with an absolute reduction in mortality of $9.2 \%$ at 15 years (38). Tamoxifen is the commonly used hormonal medicine for the management of breast cancer irrespective of menopausal status in Nepal $(36,39)$. However, tamoxifen is associated with serious adverse events (SAEs) including endometrial cancer and thromboembolic events due to its estrogen-agonistic activity in selected tissues (40). The patient's health status is vital when choosing the most appropriate chemotherapy regimen for breast cancer. An anthracycline-based chemotherapy regimen is also commonly used in Nepal (41). Ideal candidates for an anthracycline-containing regimen include women with chemotherapy naive, stage IV breast cancer and those who have not previously received an anthracycline (42). Anthracycline should be avoided in patients was cardiac history, as it may damage the myocardium through free reactive oxygen radicals and cause direct DNA damage; such patients treated with anthracycline typically develop cardiac dysfunction as a result of the treatment $(43,44)$. For combination chemotherapy, patient preferences aid to individualize the 
treatment plan as certain patients may not accept the additional risks of toxicity associated with combination chemotherapy.

The primary goal of palliative care for breast cancer is to prevent and ease suffering and improve the quality of life of women with metastatic breast disease. Bone metastases are a common cause of chronic pain, with pain resulting directly from expanding lesions, pathological fracture or damage to adjacent structures (45). Opioid analgesics are widely used to treat pain in patients with breast cancer due to their safety, multiple routes of administration, ease of titration, reliability, and effectiveness for all types of pain (46). Glucocorticoids should be considered for the inflammation or pain related to nerve compression. The National Comprehensive Cancer Network recommend nonsteroidal anti-inflammatory drugs (NSAIDs) and acetaminophen in combination with opioids for bone pain not associated with spinal cord compression or fracture (47). Bisphosphonates, glucocorticoids or systemic administration of radioisotopes should be considered for diffuse bone pain resulting from metastases $(46,47)$. Such palliative care for breast cancer in Nepal is still in its infancy $(48,49)$.

\section{Breast cancer awareness and future directions}

Studies have repeatedly demonstrated that irrespective of their socio-economic and educational backgrounds, Nepalese women had poor knowledge of the risk factors of breast cancer $(8,9,50)$. Although age, education, household income and family history of breast cancer are reported to be significantly associated with awareness level, a study on breast cancer literacy among higher secondary students demonstrated that students had poor knowledge on breast cancer risk factors, symptoms and curability (50). Furthermore, most of the students lacked awareness of age, early menarche, delayed menopause and use of the oral contraceptive pill as being the risk factors for breast cancer. Sathian et al (8) identified that among female residents of Pokhara in Nepal, the level of awareness and understanding of breast cancer, including knowledge of warning signs and BSE, was sub-optimal. Another cross-sectional study conducted at KIST medical college in Kathmandu revealed that $70 \%$ of the participants had never heard of breast cancer (9). This study demonstrated that awareness and understanding of breast cancer among Nepalese women was notably poor. Nepalese women need to be aware of modifiable and non-modifiable risk factors of breast cancer to adopt appropriate practices for prevention. Cancer literacy provided by healthcare settings should be increased to ensure this knowledge becomes more widespread amongst the general public. The government should also formulate formal policies for effective nation- and district-wide cancer literacy programmes, as well as engagements with community-level, national and international organizations and the healthcare system. Additionally, breast health education sessions, guidance by nurses on BSE, regular follow-up by nurses and other healthcare training institutions should carried out throughout the country. An awareness campaign could be conducted through advertisements on breast cancer and screening via radio, internet, television and poster platforms to promote an attitude change regarding breast cancer in women. There is also a need to strengthen the cancer-related curriculum in medical schools, with focus on breast cancer awareness and screening methods. Furthermore, future research should continue to focus on the genetic makeup of breast cancer, to further improve understanding on the early onset of breast cancer in Nepal and other regions.

\section{Conclusion}

In conclusion, the overall perspectives for breast cancer are notably variable among women in Nepal. Breast cancer has emerged as the second more prevalent malignancy in women in Nepal. Factors responsible for the general delayed diagnosis are lack of knowledge, limited breast cancer screening programs, limited health care facilities and sociocultural barriers. The government should foster novel policies to establish effective nationwide cancer literacy programmes, as well as engagements with community-level, national and international organizations and the healthcare system.

\section{Acknowledgements}

The authors are thankful to Professor Yang Lü from the Department of Geriatrics and Professor Shu Liang Guo from the Department of Respiratory Medicine of Chongqing Medical University for their help during the preparation of this article.

\section{Funding}

No funding was received.

\section{Availability of data and materials}

Not applicable.

\section{Authors' contributions}

MG and MG completed the online database search for relevant information and designed the concept. MG, MG, RJT, BU and $\mathrm{BP}$ wrote sections of the manuscript. RJT, BU and BP were involved in revision of the manuscript at all stages. All authors read and approved the final manuscript.

\section{Ethics approval and consent to participate}

Not applicable.

\section{Consent for publication}

Not applicable.

\section{Competing interests}

The authors declare that they have no competing interests.

\section{References}

1. American Cancer Society: Cancer Facts \& Figures 2018 https://www.cancer.org/content/dam/cancer-org/research/ cancer-facts-andstatistics/annual-cancer-facts-and-figures/2018/ cancer-facts-and-figures-2018.pdf. (Last accessed January 25, 2018).

2. Mariotto AB, Yabroff KR, Shao Y, Feuer EJ and Brown ML: Projections of the cost of cancer care in the United States: 2010-2020. J Natl Cancer Inst 103: 117-128, 2011. 
3. Youlden DR, Cramb SM, Yip CH and Baade PD: Incidence and mortality of female breast cancer in the Asia-Pacific region. Cancer Biol Med 11: 101-115, 2014.

4. Singh YP and Sayami P: Management of breast cancer in Nepal. JNMA J Nepal Med Assoc 48: 252-257, 2009.

5. Pun CB, Pradhananga KK, Siwakoti B, Subedi K and Moore MA: Malignant neoplasm burden in Nepal- Data from the seven major cancer service hospitals for 2012. Asian Pac J Cancer Prev 16 8659-8663, 2015.

6. Benz CC: Impact of aging on the biology of breast cancer. Crit Rev Oncol Hematol 66: 65-74, 2008.

7. Agarwal G and Ramakant P: Breast Cancer Care in India: The Current Scenario and the Challenges for the Future. Breast Care (Basel) 3: 21-27, 2008.

8. Sathian B, Nagaraja SB, Banerjee I, Sreedharan J, De A, Roy B, Rajesh E, Senthilkumaran S, Hussain SA and Menezes RG: Awareness of breast cancer warning signs and screening methods among female residents of Pokhara valley, Nepal. Asian Pac J Cancer Prev 15: 4723-4726, 2014.

9. Shrestha K: Breast cancer knowledge and screening practice among women visited to KIST medical college. Nepal Med Coll J 14: 308-311, 2012.

10. GLOBOCAN 2012: Estimated Cancer Incidence, Mortality and Prevalence Worldwide in 2012. http://globocan.iarc.fr/Pages/ fact_sheets_population.aspx (Last accessed January 25, 2018).

11. Forsea AM: Cancer registries in Europe-going forward is the only option. Ecancermedicalscience 10: 641, 2016.

12. Pradhananga KK, Baral M and Shrestha BM: Multi-institution hospital-based cancer incidence data for Nepal: An initial report. Asian Pac J Cancer Prev 10: 259-262, 2009.

13. Binu V, Chandrashekhar T, Subba S, Jacob S, Kakria A, Gangadharan P and Menezes RG: Cancer pattern in Western Nepal: A hospital based retrospective study. Asian Pac J Cancer Prev 8: 183-186, 2007.

14. Moore MA, Ariyaratne Y, Badar F, Bhurgri Y, Datta K, Mathew A, Gangadharan P, Nandakumar A, Pradhananga KK, Talukder MH, et al: Cancer epidemiology in South Asia - past, present and future. Asian Pac J Cancer Prev 11 (Suppl 2): 49-66, 2010.

15. Anothaisintawee T, Wiratkapun C, Lerdsitthichai P, Kasamesup V, Wongwaisayawan S, Srinakarin J, Hirunpat S, Woodtichartpreecha $\mathrm{P}$, Boonlikit $\mathrm{S}$, Teerawattananon $\mathrm{Y}$, et al: Risk factors of breast cancer: A systematic review and meta-analysis. Asia Pac J Public Health 25: 368-387, 2013.

16. Aich RK, Mondal NK, Chhatui B, Sepai HM, Aich R, Acharyya A, Manir K and Bhattacharaya J: Relevance of risk factors of breast cancer in women: An Eastern Indian scenario. J Cancer Res Ther 12: 302-308, 2016.

17. Collaborative Group on Hormonal Factors in Breast Cancer: Menarche, menopause, and breast cancer risk: Individual participant meta-analysis, including 118964 women with breast cancer from 117 epidemiological studies. Lancet Oncol 13: $1141-1151,2012$

18. Porter P: "Westernizing" women's risks? Breast cancer in lower-income countries. N Engl J Med 358: 213-216, 2008.

19. Bhattacharya $S$ and Adhikary S: Evaluation of risk factors, diagnosis and treatment in carcinoma breast--a retrospective study. Kathmandu Univ Med J (KUMJ) 4: 54-60, 2006. (KUMJ)

20. Singh Y, Sayami P, Sayami G, Nakagawa H, Koreeda T, Hatano T and Tsubura A: Nepalese breast cancer in relation to reproductive factors: Comparison between Nepalese and Japanese cases Anticancer Res 22 (1A): 319-323, 2002.

21. Melvin JC, Wulaningsih W, Hana Z, Purushotham AD Pinder SE, Fentiman I, Gillett C, Mera A, Holmberg L and Van Hemelrijck M: Family history of breast cancer and its association with disease severity and mortality. Cancer Med 5: 942-949, 2016

22. Gram IT, Park SY, Kolonel LN, Maskarinec G, Wilkens LR, Henderson BE and Le Marchand L: Smoking and Risk of Breast Cancer in a Racially/Ethnically Diverse Population of Mainly Women Who Do Not Drink Alcohol: The MEC Study. Am J Epidemiol 182: 917-925, 2015.

23. Tara S, Agrawal CS and Agrawal A: Validating breast self examination as screening modalities for breast cancer in eastern region of Nepal: A population based study. Kathmandu Univ Med J (KUMJ) 6: 89-93, 2008.

24. Glasziou P and Houssami N: The evidence base for breast cancer screening. Prev Med 53: 100-102, 2011.

25. Marmot MG, Altman DG, Cameron DA, Dewar JA, Thompson SG and Wilcox M: The benefits and harms of breast cancer screening: An independent review. Br J Cancer 108: 2205-2240, 2013.
26. Sidhartha TB, Thapa B, Singh Y, Sayami P and Khanal U: Mammographic diagnosis of breast carcinoma: An institutional experience. JNMA J Nepal Med Assoc 47: 62-65, 2008.

27. Madjar H: Role of Breast Ultrasound for the Detection and Differentiation of Breast Lesions. Breast Care (Basel) 5: 109-114, 2010.

28. Kelly KM, Dean J, Comulada WS and Lee S-J: Breast cancer detection using automated whole breast ultrasound and mammography in radiographically dense breasts. Eur Radiol 20: 734-742, 2010

29. Saslow D, Boetes C, Burke W, Harms S, Leach MO, Lehman CD, Morris E, Pisano E, Schnall M, Sener S, Smith RA, et al: American Cancer Society guidelines for breast screening with MRI as an adjunct to mammography. CA Cancer J Clin 5: 75-89, 2007.

30. Pradhan GB, Shrestha R, Shrestha S, Khadka P, Bhattachan CL, Lehman CD, et al: Outcome analysis of breast cancer patients treated at Nepal Medical College. Nepal Med Coll J 14: 93-95, 2012.

31. Young OE, Valassiadou $\mathrm{K}$ and Dixon M: A review of current practices in breast conservation surgery in the UK. Ann R Coll Surg Engl 89: 118-123, 2007.

32. Fujii T, Yajima R, Yamaguchi S, Yanagita Y,Fujisawa T, Hirakata T, Tsutsumi S, Asao T, Iijima M and Kuwano H: Extracapsular invasion of sentinel lymph nodes is not associated with disease recurrence in breast cancer. Int Surg 99: 305-308, 2014.

33. Kuenen-Boumeester V, Menke-Pluymers M, de Kanter AY, Obdeijn IM, Urich D and Van Der Kwast TH: Ultrasound-guided fine needle aspiration cytology of axillary lymph nodes in breast cancer patients. A preoperative staging procedure. Eur J Cancer 39: 170-174, 2003.

34. Headon H, Kasem A, Almukbel R and Mokbel K: Improvement of survival with postmastectomy radiotherapy in patients with 1-3 positive axillary lymph nodes: A systematic review and meta-analysis of the current literature. Mol Clin Oncol 5: 429-436, 2016.

35. Yang TJ and Ho AY: Radiation therapy in the management of breast cancer. Surg Clin North Am 93: 455-471, 2013.

36. Sapkota A, Shrestha S, Sedhain A, Koirala S and Kafle P: Problems of Breast Cancer Survivors Living in an Urban Area of Nepal. Asia Pac J Oncol Nurs 3: 289-296, 2016.

37. Gonzalez-Angulo AM, Morales-Vasquez F, and Hortobagyi GN: Overview of resistance to systemic therapy in patients with breast cancer. Adv Exp Med Biol 608: 1-22, 2007.

38. Early Breast Cancer Trialists' Collaborative Group (EBCTCG): Effects of chemotherapy and hormonal therapy for early breast cancer on recurrence and 15-year survival: An overview of the randomised trials. Lancet 365: 1687-1717, 2005.

39. Perez EA: Safety profiles of tamoxifen and the aromatase inhibitors in adjuvant therapy of hormone-responsive early breast cancer. Ann Oncol 18 (Suppl 8): viii26-35, 2007.

40. Karn A, Jha AK, Shrestha S, Acharya B, Poudel S and Bhandari RB: Tamoxifen for breast cancer. JNMA J Nepal Med Assoc 49: 62-67, 2010.

41. Acharya SC, Jha AK and Manandhar T: Clinical profile of patients presenting with breast cancer in Nepal. Kathmandu Univ Med J (KUMJ) 10: 3-7, 2012. (KUMJ).

42. SparanoJA, Wang M,MartinoS,Jones V,PerezEA,SaphnerT, etal: Weekly paclitaxel in the adjuvant treatment of breast cancer. N Engl J Med 358: 1663-1671, 2008

43. Romond EH, Perez EA, Bryant J, Suman VJ, Geyer CE Jr, Davidson NE, Tan-Chiu E, Martino S, Paik S, Kaufman PA, et al: Trastuzumab plus adjuvant chemotherapy for operable HER2-positive breast cancer. N Engl J Med 353: 1673-1684, 2005.

44. FDA: PLp: doxorubicin $\mathrm{HCl}$ intravenous injection, doxorubicin $\mathrm{HCl}$ intravenous injection. New York, 2013.

45. Irvin W Jr, Muss HB and Mayer DK: Symptom management in metastatic breast cancer. Oncologist 16: 1203-1214, 2011.

46. Lussier D, Huskey AG and Portenoy RK: Adjuvant analgesics in cancer pain management. Oncologist 9: 571-591, 2004.

47. National Comprehensive Cancer Network (NCCN) Adult Cancer Pain: [accessed December 30, 2016]. https://www.nccn.org/ professionals/physician_gls/pdf/pain.pdf

48. Braun $\mathrm{CM}$ and Itano $\mathrm{JK}$ : Cancer care in Nepal: variables that affect diagnosis, treatment, and prognosis: a case study. Cancer Nurs 24: 137-142, 2001.

49. Piya MK and Acharya SC: Oncology in Nepal. South Asian J Cancer 1: 5-8, 2012.

50. Bhandari PM, Thapa K, Dhakal S, Bhochhibhoya S, Deuja R, Acharya $\mathrm{P}$ and Mishra SR: Breast cancer literacy among higher secondary students: Results from a cross-sectional study in Western Nepal. BMC Cancer 16: 119, 2016. 